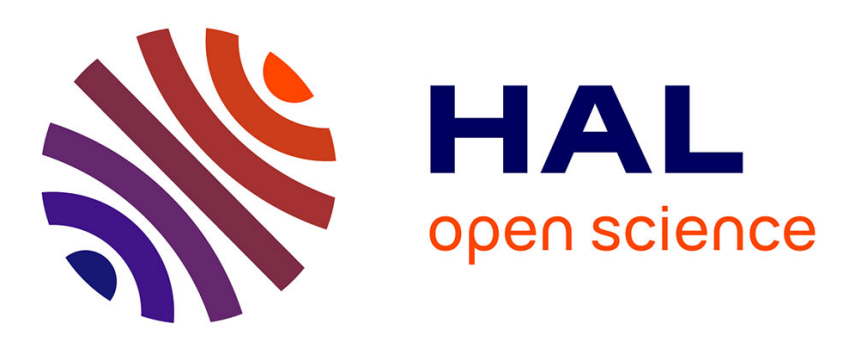

\title{
Simulation-based evaluation of a cold atom interferometry gradiometer concept for gravity field recovery
}

Karim Douch, Hu Wu, Christian Schubert, Jürgen Müller, Franck Pereira dos Santos

\section{To cite this version:}

Karim Douch, Hu Wu, Christian Schubert, Jürgen Müller, Franck Pereira dos Santos. Simulationbased evaluation of a cold atom interferometry gradiometer concept for gravity field recovery. Advances in Space Research, 2018, 61 (5), pp.1307-1323. 10.1016/j.asr.2017.12.005 . hal-01826234

\section{HAL Id: hal-01826234 \\ https://hal.sorbonne-universite.fr/hal-01826234}

Submitted on 29 Jun 2018

HAL is a multi-disciplinary open access archive for the deposit and dissemination of scientific research documents, whether they are published or not. The documents may come from teaching and research institutions in France or abroad, or from public or private research centers.
L'archive ouverte pluridisciplinaire HAL, est destinée au dépôt et à la diffusion de documents scientifiques de niveau recherche, publiés ou non, émanant des établissements d'enseignement et de recherche français ou étrangers, des laboratoires publics ou privés. 


\title{
Simulation-based evaluation of a cold atom interferometry gradiometer concept for gravity field recovery
}

\author{
Karim Douch ${ }^{\mathrm{a}, *}, \mathrm{Hu} \mathrm{Wu}^{\mathrm{a}}$, Christian Schubert ${ }^{\mathrm{b}}$, Jürgen Müller ${ }^{\mathrm{a}}$, Franck \\ Pereira dos Santos ${ }^{\mathrm{c}}$ \\ ${ }^{a}$ Institut für Erdmessung, Leibniz Universität Hannover, Schneiderberg 50, 30167 \\ Hannover, Germany \\ ${ }^{b}$ Institut für Quantenoptik, Leibniz Universität Hannover, Welfengarten 1, 30167 \\ Hannover, Germany \\ ${ }^{c}$ LNE-SYRTE, Observatoire de Paris, PSL Research University, CNRS, Sorbonne \\ Universités, UPMC Univ. Paris 06, 61 Avenue de l'Observatoire, 75014 Paris, France
}

\begin{abstract}
The prospects of future satellite gravimetry missions to sustain a continuous and improved observation of the gravitational field have stimulated studies of new concepts of space inertial sensors with potentially improved precision and stability. This is in particular the case for cold-atom interferometry (CAI) gradiometry which is the object of this paper. The performance of a specific CAI gradiometer design is studied here in terms of quality of the recovered gravity field through a closed-loop numerical simulation of the measurement and processing workflow. First we show that mapping the time-variable field on a monthly basis would require a noise level below $5 \mathrm{mE} / \sqrt{\mathrm{Hz}}$. The mission scenarios are therefore focused on the static field, like GOCE. Second, the stringent requirement on the angular velocity of a one-arm gradiometer, which must not exceed $10^{-6} \mathrm{rad} / \mathrm{s}$, leads to two possible modes of operation of the CAI gradiometer: the nadir and the quasi-inertial mode. In the nadir mode, which corresponds to the usual Earth-pointing satellite attitude, only
\end{abstract}

\footnotetext{
*Corresponding author

Email addresses: douch@ife.uni-hannover.de (Karim Douch), wuhu@ife.uni-hannover.de $(\mathrm{Hu} \mathrm{Wu})$, schubert@iqo.uni-hannover.de (Christian Schubert), mueller@ife.uni-hannover.de (Jürgen Müller), franck.pereira@obspm.fr (Franck Pereira dos Santos)
} 
the gradient $V_{y y}$, along the cross-track direction, is measured. In the quasiinertial mode, the satellite attitude is approximately constant in the inertial reference frame and the 3 diagonal gradients $V_{x x}, V_{y y}$ and $V_{z z}$ are measured. Both modes are successively simulated for a $239 \mathrm{~km}$ altitude orbit and the error on the recovered gravity models eventually compared to GOCE solutions. We conclude that for the specific CAI gradiometer design assumed in this paper, only the quasi-inertial mode scenario would be able to significantly outperform GOCE results at the cost of technically challenging requirements on the orbit and attitude control.

Keywords: Cold atom interferometry; space gravity gradiometry; gravity field recovery; closed-loop simulation

\section{Introduction}

The GOCE (Rummel et al., 2011) and GRACE (Tapley et al., 2004) missions have provided unprecedented insights in the static and time-variable gravitational field of the Earth and proved to be very useful in numerous fields, from unification of height systems to the determination of global mass distribution and mass transport in the Earth system (Kusche et al., 2012).

The two satellites of the gravimetry mission GRACE (Gravity Recovery and Climate Experiment) are orbiting the Earth since 2002. Its major measurement method relies on Satellite-to-Satellite ranging which measures the varying distance between the two satellites with $\mu \mathrm{m}$ precision using a microwave tracking system. GRACE provided monthly solutions of the gravity field during most of its lifetime and therefore enables analysing its temporal variations. The latter are caused, among others, by ice mass loss in Greenland or Antarctica, glacial isostatic rebound or changes in the global hydrological cycle see, e.g. Eicker et al. (2016). The GOCE (Gravity field and steadystate Ocean Circulation Explorer) mission delivered data between 2009 and 2013, which allowed to determine the geoid (Torge and Müller, 2012) with an accuracy of about 1 to $2 \mathrm{~cm}$ for a spatial resolution of $100 \mathrm{~km}$ on the Earth surface. This provided the basis to establish global physical height systems (i.e. those related to the gravity field) and - combined with altimetric measurements of the mean sea surface - to estimate global ocean currents. GOCE is also the first mission which employed gradiometry. The core of the GOCE gradiometer consists of 3 pairs of electrostatic accelerometers measuring the differential gravitational accelerations on its sensitive axis with a 
noise of about $10 \mathrm{mE} / \sqrt{\mathrm{Hz}}\left(1 \mathrm{E}=10^{-9} \mathrm{~s}^{-2}\right)$ in a measurement bandwidth (MBW) between 5 and $100 \mathrm{~Hz}$ (Rummel et al., 2011). Hereafter, the noise level will be expressed (if not mentioned otherwise) in terms of amplitude spectral density (ASD) which is the square root of the power spectral density.

Novel science results were obtained thanks to GRACE and GOCE, giving insight in a multitude of processes in the hydrosphere, cryosphere, oceans, atmosphere and solid Earth that could not be assessed before. As stressed in various reports as for instance in Pail et al. (2015) or Panet et al. (2013), there is a strong demand for sustaining a global, homogeneous and continuous observation of the Earth's gravitational field. Furthermore, the spatial and temporal resolution along with the precision should be improved in order to meet the challenging requirements of the different end users. Therefore, new concepts for follow-on missions have been developed with the aim to achieve a better resolution (ideally below $100 \mathrm{~km}$ spatially and below 1 month temporally), a higher accuracy and a longer time series (Gruber and Team, 2014; Elsaka et al., 2014).

In this paper we investigate to what extent a new concept of gradiometer based on cold-atom interferometry (CAI) sensors (Carraz et al., 2014) can reach such objectives. Gradiometry offers several advantages: a gradiometer measures directly a functional of the gravitational field, moreover, it performs like a high-pass filter increasing the relative sensitivity to the high-frequencies of the gravitational signal corresponding to the small wavelengths of the field. The constraints for the measurement bandwidth of a CAI gradiometer might be relaxed and potentially a higher accuracy might be achieved. Last but not least, CAI technology provides a very good stability of the gradiometer bias and scale factor, a high common mode rejection of $140 \mathrm{~dB}$ was demonstrated by McGuirk et al. (2002) and is likely to have white noise over a wide frequency range while the GOCE gradiometer noise was approximately white only in its MBW.

After a brief description of the physical principle of the gradiometer concept and the conditions to operate it in space, the method of evaluation of its performance in terms of quality of the recovered gravity field model is described. We have chosen to carry out the study through a closed-loop simulation of the measurement process in the time domain. Finally the results and the technical feasibility of the proposed concept are discussed. 


\section{Principle of the CAI gradiometer concept}

The gravity gradients $V_{i j}$ are the second-order partial derivatives of the gravitational potential $V$ :

$$
V_{i j}=\frac{\partial^{2} V}{\partial i \partial j}
$$

where $x_{i}$ and $x_{j}$, are Cartesian coordinates of the 3 dimensional space. The resulting 9 components give the GGT (gravitational gradient tensor). In the following, we note $\mathbf{V}$ the GGT and $V_{i j} i, j \in\{x, y, z\}$ any component of the tensor. Since the gradiometer is an inertial sensor, it is not possible in a non-inertial frame to measure directly the gravitational gradients, but rather the gradients of the acceleration field. The latter are however connected to the gravitational gradients as follows (for the diagonal elements):

$$
\begin{aligned}
& V_{x x}=\Gamma_{x x}-\omega_{z}^{2}-\omega_{y}^{2} \\
& V_{y y}=\Gamma_{y y}-\omega_{z}^{2}-\omega_{x}^{2} \\
& V_{z z}=\Gamma_{z z}-\omega_{x}^{2}-\omega_{y}^{2}
\end{aligned}
$$

where $\Gamma_{i i} i \in\{x, y, z\}$ is the diagonal acceleration gradient along the $i$-axis and $\left(\omega_{x}, \omega_{y}, \omega_{z}\right)^{t}=\Omega$ is the vector of the angular velocity of the GRF (gradiometer reference frame) with respect to the IRF (inertial reference frame). We will refer hereafter to the terms in $\omega$ in equations (2) as the centrifugal terms. Finally, two other observables must be given in order to define correctly the gravitational gradient: the position of the measurement point and the orientation of the measurement frame, i.e. the attitude of the GRF with respect to the IRF.

In the case of the CAI gradiometer concept presented here, the acceleration gradient is approximated by the difference of acceleration measured simultaneously at adjacent points. For the $x$ axis, one has for instance

$$
\Gamma_{x x}=\frac{\Delta a_{x}}{l}
$$

where $\Delta a_{x}$ is the difference of the x-component of the acceleration between two points aligned along the $\mathrm{x}$-direction and separated by a baseline $l$. Similar expressions hold for the two other diagonal acceleration gradients.

Here we introduce the light-pulse atom interferometry utilized in the acceleration measurement and the CAI gradiometer considered in this study 


\section{Beam splitting light field}
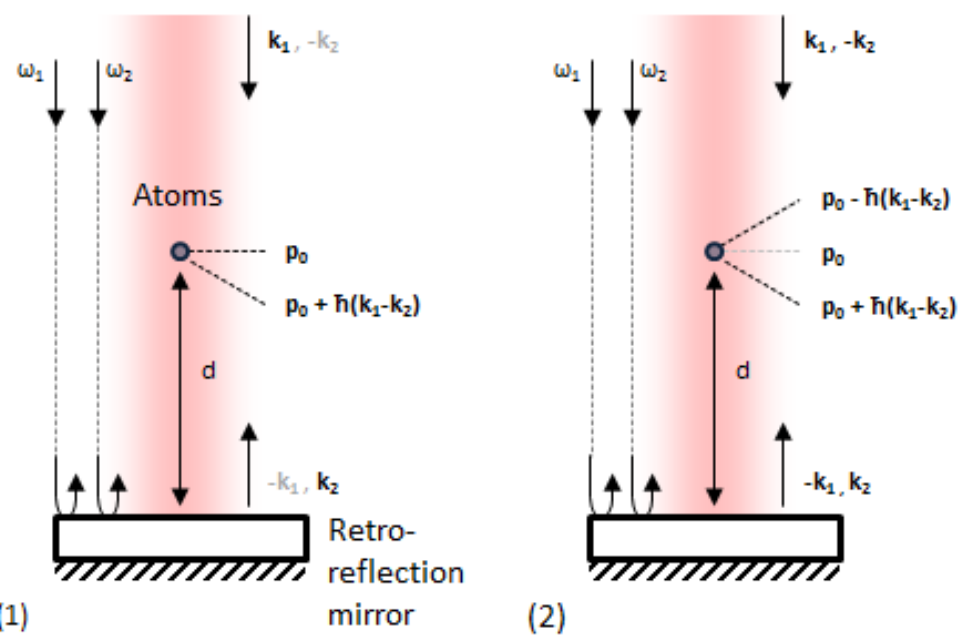

(2)

Figure 1: Atom-light interaction for beam splitting. (1) The laser beam features two frequencies $\omega_{1}, \omega_{2}$ and is retro reflected at the mirror. This leads to four different components in the light, distinguished by frequency and / or propagation direction $(\mathbf{k})$. Depending on the duration of the interaction of the light field with the atoms, the atoms are coherently excited into a superposition state of initial momentum $\mathbf{p}_{0}$ and $\mathbf{p}_{0}+\hbar\left(\mathbf{k}_{1}-\mathbf{k}_{2}\right)$ ( $\hbar$ reduced Planck's constant) by absorption and stimulated emission of a photon from the incoming (wave vector $\mathbf{k}_{1}$ ) and the retro reflected beam (wave vector $\mathbf{k}_{2}$ ). During this process, the phase $\mathbf{k} \cdot \mathbf{d}$ is imprinted onto the atoms, depending on the distance of the atoms to the mirror $d$ and the effective wave vector $\mathbf{k}=\mathbf{k}_{1}-\mathbf{k}_{2}$. (2) In the special case of negligible velocity of the atom parallel to the effective wave vector $\mathbf{k}$, a four photon process leads to a symmetric momentum transfer with the superposition of the three momentum states $\mathbf{p}_{0}$ and $\mathbf{p}_{0} \pm \hbar\left(\mathbf{k}_{1}-\mathbf{k}_{2}\right)$, effectively doubling the effective wave number. 


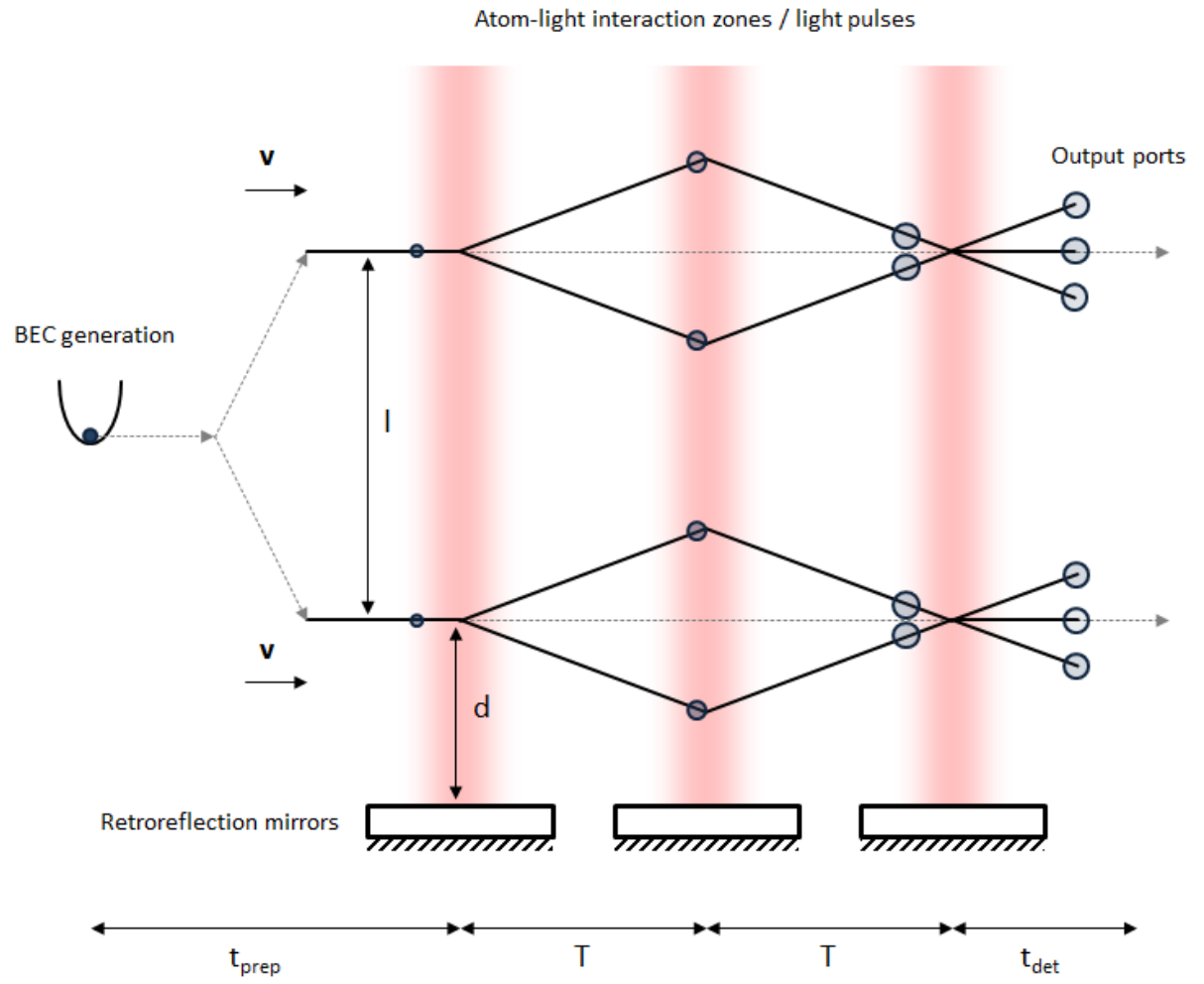

Figure 2: Atom interferometry scheme for gradiometric measurements as proposed in (Carraz et al., 2014). During the preparation time $t_{\text {prep }}$ an atomic ensemble is generated, split into two leading to a separation 1 between the ensembles, and injected into the two atom interferometers with forward drift velocity $\mathbf{v}$. Here, the atomic ensembles are in free fall. The three-pulse sequence with drift times $\mathrm{T}$ forms a diamond shaped geometry. The first pulse imprints a reference phase $(\mathbf{k} \cdot \mathbf{d})$ onto the atoms to which the phases imprinted at the two subsequent pulses is compared. In a simplified picture, the total phase accumulates to $\mathbf{k d}(0)-2 \mathbf{k d}(T)+\mathbf{k} \mathbf{d}(2 T)$, where $\mathbf{d}(t)$ denotes the centre of wave packet position (grey dotted line) and other spurious contributions are omitted. With $\mathbf{d}(t)=\mathbf{d}_{0}+\mathbf{v}(t)+\frac{1}{2} \mathbf{a} T^{2}$ the phase shift becomes $\mathbf{k} \cdot \mathbf{a} T^{2}$. This result is recovered for the upper interferometer with the replacement $\mathbf{d} \rightarrow \mathbf{d}+\mathbf{l}$. Differential displacements $\left(\mathbf{d}_{0}=\mathbf{d}_{1} \neq \mathbf{d}_{2} \neq \mathbf{d}_{3}\right)$ or vibration of the mirrors $(1-3)$ are suppressed in the differential signal. Depending on the total phase shift, the transition probability oscillates between the inner an the outer output port. During detection, this transition probability is recorded by flashing light onto the atoms and either collecting the fluorescence or measuring the absorption. This configuration enables a measurement in 1D and can be reproduced for the other two axis for a 3D measurement. A preparation time $t_{\text {prep }}$ shorter than the total interferometer time $2 T$ enables an interleaved mode of operation (Carraz et al., 2014). 
which was proposed in (Carraz et al., 2014), based on recent developments in microgravity experiments (Barrett et al., 2016; Rudolph et al., 2015; Aguilera et al., 2014; Müntinga et al., 2013). An atom interferometer exploits interference of matter waves which are coherently manipulated by light fields. At each atom-light interaction, a phase is imprinted onto the atoms which depends on the relative position between the light field and the atoms (see 1). The light field, consisting of two frequencies, is retro reflected at a mirror and drives two-photon transitions during each interaction. This couples different momentum states which are required to form the interferometer geometry sensitive to accelerations. Effectively, the mirror serves as a reference plane for the position of the atoms during the interactions. A typical implementation for an atom interferometer sensitive to accelerations is a three pulse sequence as used in various experiments (Peters et al., 1999; McGuirk et al., 2002; Louchet-Chauvet et al., 2011; Hu et al., 2013; Rosi et al., 2014; Kovachy et al., 2015a; Berg et al., 2015; Freier et al., 2016; Abend et al., 2016) and also proposed for the CAI gradiometer (Carraz et al., 2014) as shown in fig. 2. Initially, a beam splitter pulse creates a coherent superposition between two momentum states. Due to the double diffraction (Lévèque et al., 2009) employed here, the trajectories drift apart with four single photon momenta. After a free evolution time $T$, a second pulse coherently inverts the momenta. This allows closing the atom interferometer with a third beam splitting pulse after another free evolution time $T$. The detection reads out the relative population of the output ports. More elaborated schemes, spatially resolving structures in the atomic ensembles are available (Dickerson et al., 2013; Müntinga et al., 2013), but in the most simple case, the populations $N_{1}, N_{2}$ of the two ports are just counted. The normalized output signal then reads

$$
P\left(\phi_{a}\right)=N_{1} /\left(N_{1}+N_{2}\right)=P_{0}+A \cdot \cos \left(\phi_{a}\right)
$$

with offset $P_{0}$, amplitude $A$, contrast $C=A / P_{0}$, and accumulated phase $\phi_{a}$. Neglecting other effects, the low expansion rate enables high beam splitting efficiency, and consequently a contrast close to unity $C \sim 1$. Neglecting finite pulse durations (Antoine, 2007), and omitting other / higher order terms, the phase shift due to accelerations a is calculated (Bordé, 2004; Arimondo et al., 2009) to

$$
\phi_{a}=\mathbf{k} \cdot \mathbf{a} T^{2}
$$


where $\mathbf{k}$ denotes the effective wave vector corresponding to the four photon momenta. Here, the three-pulse sequence is preferred to the four-pulse sequence also discussed in (Carraz et al., 2014), because it offers a higher contrast and a larger scale factor, scaling as $\sim T^{2}$.

Additional elements important for a light-pulse atom interferometer are the source generating the atomic ensemble, and the detection system, reading out the output ports of the atom interferometer. For each cycle, the source prepares an atomic ensemble with a number of atoms $N$, and a finite velocity spread $\sigma_{v}$ which corresponds to an (effective) temperature. In CAI (Carraz et al., 2014), the generation of ${ }^{87} R b$ Bose-Einstein condensates is proposed for the source to enable very low residual expansion rates at a level of $100 \mu \mathrm{m} / \mathrm{s}$ (Kovachy et al., 2015b; Müntinga et al., 2013).

The fundamental limit in such an interferometer is the quantum projection noise (QPN), implying a minimum phase noise per cycle of

$$
\sigma_{\phi_{Q P N}}=1 /(C \sqrt{N})
$$

Consequently, the QPN limited acceleration sensitivity is $\sigma_{a}=\frac{1}{C \sqrt{N} k T^{2}} \sqrt{\frac{t_{c}}{\tau}}$ for a cycle time $t_{c}$, an integration time $\tau, k=|\mathbf{k}|$, and acceleration a parallel to $\mathbf{k}$. Manipulating two of these atom interferometers, spatially separated by a baseline $l$, simultaneously with the same beam splitter light fields forms the gradiometer geometry (McGuirk et al., 2002). The gradient information is recovered from the differential signal divided by the baseline. In this case, the QPN limited sensitivity to an acceleration gradient is

$$
\sigma_{\Gamma}=\frac{\sqrt{2}}{C \sqrt{N} k T^{2} l} \sqrt{\frac{t_{c}}{\tau}}
$$

for an acceleration gradient $\Gamma$ in direction of the beam splitters, $\mathbf{k} \cdot \mathbf{l}=k l$. In (Carraz et al., 2014), an interleaved measurement mode is proposed. Several atom interferometers are operated concurrently with a cycle time $t_{c}=1 \mathrm{~s}$. With $N=10^{6}, k=8 \pi /(780 \mathrm{~nm}), T=5 \mathrm{~s}$, and $l=0.5$, a sensitivity of $3.5 \mathrm{mE} / \sqrt{\mathrm{Hz}}$ is obtained by Carraz et al. (2014), which corresponds to a white noise with a one-sided ASD of $3.5 \times \sqrt{2} \approx 5 \mathrm{mE} / \sqrt{\mathrm{Hz}}$.

In principle, the trace of the gradient tensor can be measured by reproducing this set-up in all three axes. In a nadir orientation, an additional constraint has to be considered. A typical GOCE-like altitude would imply an orbital frequency of $\Omega \sim 1 \mathrm{mrad} / \mathrm{s}$ which affects the interferometers 
oriented along track and in the nadir direction. Due to the rotation, the trajectories do not close anymore at the third beam splitting pulse, implying a significant contrast reduction. The two trajectories will miss each other by $\sim 1.4 \mathrm{~mm}$ (in direction of forward drift velocity $\mathbf{v}$ ) which is significantly larger than the thermal de Broglie wavelength of the atoms of $\sim 19 \mu \mathrm{m}$. Without sufficient overlap, the contrast is lost, rendering the gradiometer insensitive. Due to the linear dependency, a reduction of the rotation rate by a factor of $\sim 1000$ would be required to recover the contrast. Additionally, a rotation induced phase shift $\phi_{\text {rot }}=2(\mathbf{k} \times \mathbf{v}) \cdot \Omega T^{2}$ appears if a drift velocity $\mathbf{v}$ is present. In the CAI proposal, the atoms are launched into the interferometer with a forward drift velocity $v \approx 2.4 \mathrm{~cm} / \mathrm{s}, v=|\mathbf{v}|,|\mathbf{k} \times \mathbf{v}|=k v$. This shift is cancelled in the differential signal between the accelerometers which contains the information of the gradient, but only if the forward drift velocities are the same for both. Due to the finite velocity spread and the finite number of atoms per ensemble, a random velocity uncertainty of $\sigma_{v} / \sqrt{N}$ which is uncorrelated between the interferometers will remain. This limits the maximum rotation rate compatible with shot noise limited performance to $\sim 1 \mu \mathrm{rad} / \mathrm{s}$, including some margin. This value is similar to the requirement for closing the interferometer. Moreover, the finite velocity distribution of the ensemble implies a velocity dependent dephasing. When averaging over an output port by counting the population, the signal vanishes $(C \rightarrow 0)$, since a fringe pattern inside the output port appears for a rotation rate significantly exceeding $\sim 1 \mu \mathrm{rad} / \mathrm{s}$ leading to $2\left(k \cdot \sigma_{v}\right) \cdot \Omega T^{2} \gg 0.1 \pi(($ Tackmann et al., 2012$))$. Without further modifications, a maximum rotation rate of $\sim 1 \mu \mathrm{rad} / \mathrm{s}$ can only be maintained in the cross-track direction if a nadir pointing mode is chosen. Counter rotating the mirror to cancel the rotation rate (Freier et al., 2016; Dickerson et al., 2013; Lan et al., 2012) is not an option due to the interleaved mode (several concurrent atom interferometers) and the huge dynamic range requirement of several tens of milli-radians. Note, that a gravity gradient parallel to $\mathbf{k}$ also leads to a non-closed atom interferometer, where the displacement at the last beam splitter pulse is parallel to $\mathbf{k}$. This effect can be mitigated as described in (Roura et al., 2014; Roura, 2017). An alternative is the operation in a quasi-inertial pointing mode with maximum spurious rotation rates of $1 \mu \mathrm{rad} / \mathrm{s}$ in all three axes. These two pointing modes, nadir and quasi-inertial, motivate the performance discussion in the following sections. 


\section{Framework and method of the simulation}

\subsection{Objectives of a potential CAI gradiometer mission}

Before starting any simulation, it is important to clearly specify the scientific objectives of a potential CAI gradiometer mission, keeping in mind the expected noise level of the sensor. These objectives will be a key factor in the choice of the satellite orbit which is an essential parameter of the simulation study. As previously stated in the introduction, the various proposals for future space gravity missions emphasize the need to sustain a continuous monitoring of the time-variable gravity field. However, and as already noticed in Carraz et al. (2014), a CAI gradiometer in the configuration described in the previous section is not sensitive enough to detect the corresponding signals with a sufficient signal-to-noise ratio (SNR) on a monthly basis.

To get an idea of the magnitude of the time-variable gravity signal as compared against the CAI gradiometer sensitivity, the amplitude spectral densities of the time-variable $V_{x x}, V_{y y}$ and $V_{z z}$ in the orbital frame of a circular orbit at $259 \mathrm{~km}$ altitude are plotted in Figure 4 together with the expected gradiometer sensitivity. The time-variable gravitational signal is computed from one month (April 2006) of the updated Earth System Model from ESA (Dobslaw et al., 2015), which contains the contribution of the atmosphere, oceans, cryosphere, land hydrology and solid Earth. The x, y and z-direction defining the gradiometer reference frame are outlined in Figure 3. One can see that a sensitivity below $0.1 \mathrm{mE} / \sqrt{\mathrm{Hz}}$ is required if these gradients are to be detected with an SNR larger than 1 in the frequencies between $10^{-3}$ and $10^{-2} \mathrm{~Hz}$.

We have therefore decided to focus our study on the ability of a CAI gradiometer mission to recover the static gravity field. As such, the GOCE mission represents a perfect benchmark to evaluate the performance of the CAI gradiometer concept and to quantify precisely how the latter can improve our knowledge of the static field. It has thus been decided to choose for the simulations a GOCE-like orbit so that, in addition to the true error com-

putation in the closed-loop simulations, a comparison of the GOCE-derived gravity models and the ones derived from the simulated data can be carried out. 


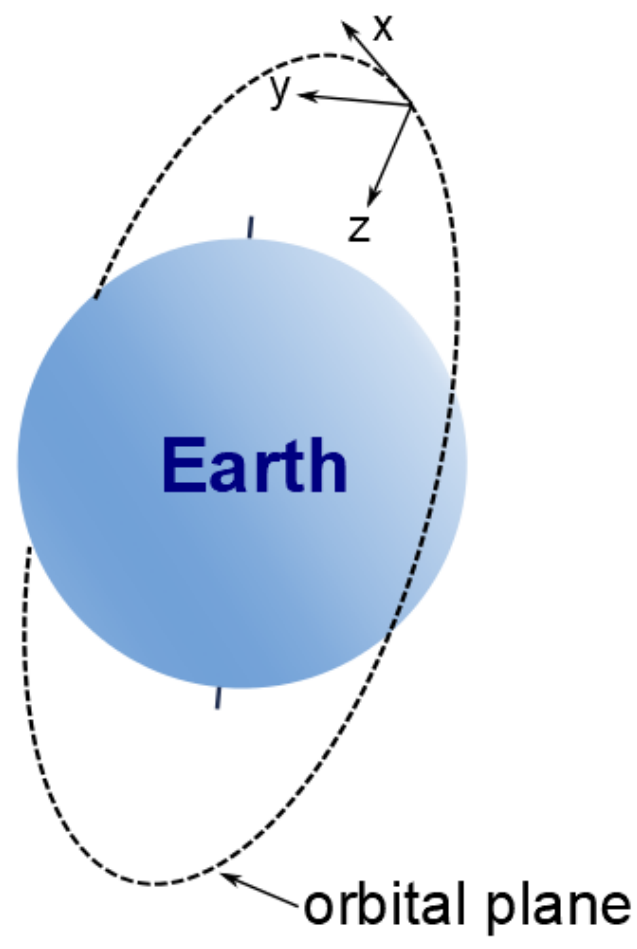

Figure 3: Orientation of the axes of the gradiometer reference frame for a nadir pointing mode. The $\mathrm{x}$-axis is parallel to the along-track direction, the $\mathrm{y}$-axis is perpendicular to the orbital plane while the z-axis points in the nadir direction.

\subsection{Description of the closed-loop simulator}

The main results of this study are based on the output of a closed-loop simulator designed in MATLAB, which enables to synthesise realistically degraded observables, namely the estimated orbit, the satellite angular velocity and attitude and the gravity gradients from which the gravitational model is finally derived. The same approach has been used for both the nadir and quasi-inertial mode, with different processing in what concerns the attitude. Starting from a global gravity field model and an orbit sampled at $1 \mathrm{~Hz}$, the GGT is computed at each measurement point in the LNOF (Local North Oriented Frame). Then the GGT is rotated into the gradiometer Reference Frame which axes define the measurement axes of the gradiometer. This requires to know for each time the true attitude of the GRF with respect to a reference frame, say the IRF. In the third step, the GGT components 


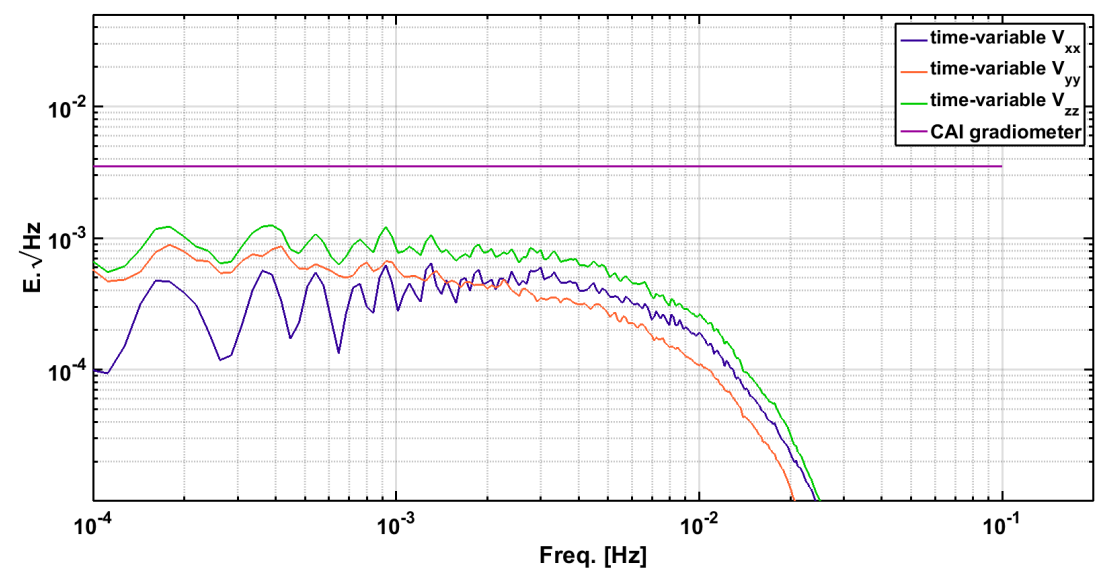

Figure 4: Comparison of the Amplitude spectral density of the expected CAI gradiometer noise to the time-variable signal in $V_{x x}, V_{y y}$ and $V_{z z}$ in the orbital frame of a circular orbit at $259 \mathrm{~km}$ altitude.

( $V_{y y}$ in the nadir case, the three diagonal elements in the quasi-inertial case) are degraded by adding the gradiometer noise and error terms due to the imperfect correction of the centrifugal part. This step also requires to have a realistic model of the estimation error of $\Omega$. Based on the noisy $\Omega$, the attitude quaternions of the GRF with respect to the IRF are computed and transformed into rotation matrices. Combined with the noisy gradients and the satellite estimated position, these data are used in a Least-Squares (LS) adjustment (see section 3.3) to recover gravity field models which are eventually compared to the initial one.

In this study, we have considered that the different observables were only corrupted by additive noise, that is we assumed a white noise floor of the sensors, as it would be the case for a solely shot-noise limited atom interferometer, ideal suppression of spurious accelerations between the two atom interferometers in gradiometer configuration, and neglected higher order terms.

\subsection{Gravity field recovery}

As the comparison is done at the level of gravity field models, we briefly describe the method used to recover them from the simulated gravity gra-

dients. The global gravity field models are usually expressed in terms of 
Spherical Harmonic (SH) series (Hofmann-Wellenhof and Moritz, 2006):

$V(r, \theta, \lambda)=\frac{G M}{R} \sum_{n=0}^{N}\left(\frac{R}{r}\right)^{n+1} \sum_{m=0}^{n}\left[\bar{C}_{n m} \cos (m \lambda)+\bar{S}_{n m} \sin (m \lambda)\right] \bar{P}_{n m}(\cos \theta)$,

where $V$ is the gravitational potential, $G M$ is the gravitational constant of the Earth, $R$ is the equatorial radius of the reference ellipsoid, $(r, \theta, \lambda)$ are the spherical coordinates ( $r$ radius, $\theta$ co-latitude, $\lambda$ longitude), $n, m$ denote SH degree and order, $N$ denotes the maximum degree of the model expansion, $\bar{P}_{n m}(\cos \theta)$ are the fully normalized associated Legendre functions, and $\bar{C}_{n m}, \bar{S}_{n m}$ are the normalized SH coefficients, also known as Stokes coefficients, which are the unknowns of the gravity field solution.

The analytical expression of the GGT $\mathbf{V}_{L N O F}$ in the local north-oriented frame can then be directly derived from equation (8). However the gravity gradients are measured in the GRF. It is therefore necessary to bring the measured gravitational gradients and the Earth's gravity field model in the same reference frame. Therefore we rotate the analytical expression of the gravitational gradients from the LNOF to the GRF. The rotation is given by:

$$
\mathbf{V}_{G R F}=\mathbf{R V}_{L N O F} \mathbf{R}^{t}
$$

where $\mathbf{R}$, the rotation matrix between the two reference frames is computed from the attitude quaternions. To resolve the observational equation, the Least-Squares (LS) adjustment method is applied. Concerning the stochastic model, a unity matrix is used mostly because white noise is assumed in the simulation. But in some cases, the spectral density of the post-fit residuals exhibits a coloured behaviour, see more details in Section 5. In such cases, a full variance/covariance matrix $(\mathrm{VCM})$ of observations is considered in the LS adjustment. The VCM is estimated from the post-fit residuals and further treated as the empirical stochastic model in an iterative step. Due to the large dimension, it is impossible to compute and store a complete VCM for the whole data set. Alternatively, the VCM is computed arc-wisely, with an arc length of 15 minutes.

Because of the orbit inclination of $96.5^{\circ}$, there is no observation in both polar regions. This is known as the polar gap problem (Sneeuw and Van Gelderen, 1997). The polar gap will severely affect the quality of the zonal and nearzonal SH coefficients (orders around 0). These coefficients are therefore omitted in the computation of the error degree variances in the rest of the study. 


\section{Description of the simulation data}

\subsection{Generation of noise-free data}

\subsubsection{Orbit and gradients in the LNOF}

As the magnitude of the high-degree harmonics of the gravitational gradients decreases rapidly with the altitude, it is crucial to have an altitude as low as possible. For the sake of rigorous comparisons to GOCE, we have chosen 71 days of the GOCE orbit starting from the $2^{\text {nd }}$ of March 2013. During this period the circular orbit had an average altitude of $239 \mathrm{~km}$ and a repeat cycle of 65 days. It must be pointed out that this altitude is lower than the altitude that GOCE had most of its lifetime which was typically of $255 \mathrm{~km}$. The time series of the orbit coordinates have been interpolated at 1 $\mathrm{Hz}$ from the GOCE L2 dynamical orbit data sampled at $0.1 \mathrm{~Hz}$ using spline functions. The static GGT expressed in the LNOF is computed using the EIGEN-6c4 model Förste et al. (2014) up to degree/order 360.

\subsubsection{Satellite angular velocity}

Nadir mode. The generation of the angular velocity $\Omega$ is split into two steps: first, by definition of the nadir mode, the instantaneous angular velocity $\omega_{\text {lorf }}$ of the local orbital frame with respect to the IRF has been computed from the orbital position and velocity and then assigned to the component $\omega_{y}$ so that the main rotation of the GRF at the orbital frequency is indeed about the $y$-axis. Second, a residual zero-mean angular velocity $\delta \Omega=\left(\delta \omega_{x}, \delta \omega_{y}, \delta \omega_{z}\right)^{t}$ is added so that we finally have:

$$
\boldsymbol{\Omega}=\left(\begin{array}{c}
\delta \omega_{x} \\
\omega_{l o r f}+\delta \omega_{y} \\
\delta \omega_{z}
\end{array}\right)
$$

This residual angular velocity consists of five successive harmonics of the orbital frequency with random phases and a 1/f decreasing amplitude, meeting the constraint of a total angular velocity perpendicular to the GRF y-axis smaller than $10^{-6} \mathrm{rad} / \mathrm{s}$.

Quasi-inertial mode. Following the definition of a truly inertial mode and keeping in mind the non-perfect attitude control and the notation used in the previous paragraph, the angular velocity vector of the GRF with respect to the IRF only consists of tiny residuals: $\Omega=\left(\delta \omega_{x}, \delta \omega_{y}, \delta \omega_{z}\right)^{t}$. However, we will see in section 5.2 that a better gravitational model can be recovered 
if the GRF rotates together with the orbital plane. In the typical case of a sun-synchronous orbit, this additional angular velocity is below $2 \times 10^{-7}$ $\mathrm{rad} / \mathrm{s}$, still within the margin of the requirement when added to a spurious rotation rate of $10^{-6} \mathrm{rad} / \mathrm{s}$. If we note $\boldsymbol{\Omega}^{\mathbf{o p}}=\left(\omega_{x}^{o p}, \omega_{y}^{o p}, \omega_{z}^{o p}\right)^{t}$ the angular velocity of the orbital plane with respect to the IRF, expressed in the GRF, we obtain:

$$
\boldsymbol{\Omega}=\left(\begin{array}{c}
\omega_{x}^{o p}+\delta \omega_{x} \\
\omega_{y}^{o p}+\delta \omega_{y} \\
\omega_{z}^{o p}+\delta \omega_{z}
\end{array}\right)
$$

This is the reason why it is more appropriate to call this mode the quasiinertial mode than the inertial mode.

\subsubsection{Attitude quaternions}

Rotations and attitude are computed using the quaternion formalism. The attitude of the GRF with respect to the inertial frame is calculated directly from the angular velocity described above and an initial condition by integrating the following differential equation:

$$
\dot{\mathbf{q}}=\frac{1}{2} \Omega \mathbf{q}
$$

where $\mathbf{q}$ is a unit quaternion describing the rotation and $\Omega$ is the angular velocity matrix of the frame. This equation has been numerically integrated with the method given in Zupan and Saje (2011). For the nadir mode, the attitude is initialized with the quaternions describing the coordinates of the local orbital frame at the initialization time. This way we make sure that the satellite attitude will be a classical Earth-pointing attitude. To avoid any possible divergence of the error of the numerical integration, the attitude is reinitialized every day. This induces a tiny jump in the attitude at midnight but it has no impact on the results of the simulations.

For the quasi-inertial mode, a question arises: is there a best quasi-inertial attitude to recover the gravitational field? For the simulations we have decided to keep an attitude for which one axis, namely the y-axis, is always perpendicular to the orbital plane so that the $V_{y y}$ gradient will be the same as in the nadir mode. Therefore the initial quaternions are chosen so that the $y$-axis is perpendicular to the orbital frame at the initialization time. 


\subsection{Generation of noisy observables}

\subsubsection{Error of the orbit determination}

For the orbit determination, we assume a zero-mean, normally distributed error with a standard deviation of $2.6 \mathrm{~cm}$ added to the orbit inertial coordinates. This precision was typically achieved with GOCE (Bock et al., 2011).

\subsubsection{Noise of the angular velocity and attitude quaternions}

For the simulation we assume that the satellite payload includes three star-trackers and a gyrometer for the determination of the angular velocity. Both kinds of sensor give estimates of the three components of the angular velocity. Star-trackers and gyrometer outputs are optimally combined at the level of angular velocity and in the frequency domain using a Wiener filter like in the processing of the angular velocity of GOCE (see Stummer et al. (2011)). This method requires to have a model of the power spectral density (PSD) of the noise of both sensors. Thereafter, we assume we have an exact knowledge of their spectral characteristics. The gyrometer considered here is the space fiber-optic gyrometer ASTRIX ${ }^{\circledR} 200$ manufactured by Airbus Defence \& Space. We considered a white noise of $6.8 \times 10^{-6} \mathrm{rad} / \mathrm{s} / \sqrt{\mathrm{Hz}}$, as guaranteed by the constructor. For the star-trackers, we assumed that the noise PSD for the three axis follows the model derived in Stummer et al. (2011) for the y-component of the angular velocity for GOCE. Both noise spectra are plotted in Figure 5 along with the example of the spectrum of a combined solution. Below $4 \times 10^{-5} \mathrm{~Hz}$ the angular velocity information is essentially given by the star-trackers while above it is only the gyrometer. The estimated attitude quaternions are deduced from the noisy angular velocity by integrating equation (12).

\subsubsection{Noise on the gradients in the GRF}

The diagonal gravitational gradients are deduced from equations (2). The final error on the gravitational gradients is therefore the sum of the gradiometer noise and the error due to the correction of the centrifugal terms. The expression of the error $\delta V_{x x}$ is given in equation (13) for the term $V_{x x}$, where the measured quantities are topped with a $\sim$ :

$$
\begin{aligned}
\tilde{V}_{x x} & =\tilde{\Gamma}_{x x}-\tilde{\omega}_{z}^{2}-\tilde{\omega}_{y}^{2} \\
& =\left(V_{x x}+\omega_{z}^{2}+\omega_{y}^{2}+n_{\Gamma}\right)-\left(\omega_{z}+n_{\omega_{z}}\right)^{2}-\left(\omega_{y}+n_{\omega_{y}}\right)^{2} \\
& =V_{x x} \underbrace{-2 \omega_{z} n_{\omega_{z}}-2 \omega_{y} n_{\omega_{y}}-n_{\omega_{z}}^{2}-n_{\omega_{y}}^{2}+n_{\Gamma}}_{\delta \mathbf{V}_{\mathbf{x x}}}
\end{aligned}
$$




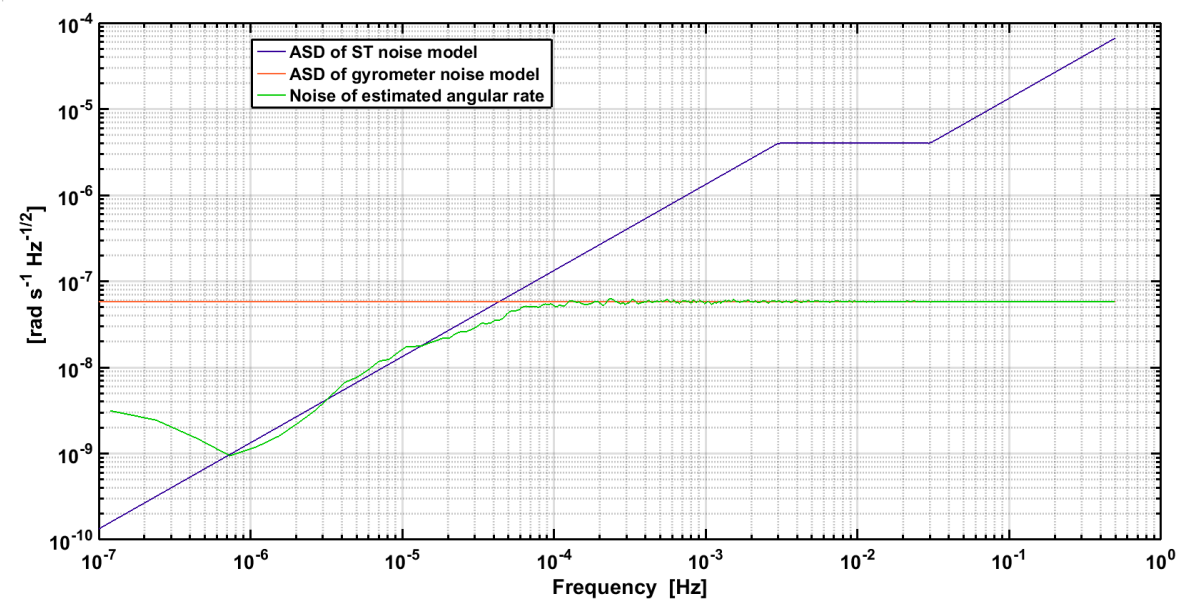

Figure 5: Amplitude spectral density of the noise degrading the estimation of one component of the angular velocity of the GRF with respect to the inertial frame.

where $n_{\omega_{y}}$ (resp. $n_{\omega_{z}}$ ) is the additive noise on $\omega_{y}$ (resp. $\omega_{z}$ ) discussed in the previous section and $n_{\Gamma}$ is the gradiometer white noise. Identical expressions can be derived for the two other gradients, for the nadir and the quasi-inertial mode.

One must also pay attention to the impact of the error of the attitude determination on the estimated gravitational gradients. We can show for analytical purposes that the additional error ${ }^{a t} \delta \mathbf{V}$ on the GGT caused by the attitude determination error is given at first order by the following expression:

$$
{ }^{a t} \delta \mathbf{V}=d \mathbf{\Theta} \mathbf{V}+\mathbf{V} d \boldsymbol{\Theta}^{t}
$$

where $\mathbf{V}$ is the GGT expressed in the GRF and $d \boldsymbol{\Theta}$ is the skew-symmetric matrix of the attitude error.

In particular, we have for the diagonal gradients:

$$
\begin{gathered}
{ }^{a t} \delta V_{x x}=-2 d \theta_{z} V_{x y}+2 d \theta_{y} V_{x z} \\
{ }^{a t} \delta V_{y y}=2 d \theta_{z} V_{x y}-2 d \theta_{x} V_{y z} \\
{ }^{a t} \delta V_{z z}=-2 d \theta_{y} V_{x z}+2 d \theta_{x} V_{y z}
\end{gathered}
$$

where $d \theta_{x} d \theta_{y}$ and $d \theta_{z}$ are the errors of the Euler angles describing the rotation between LNOF and GRF.

For the sake of simplicity, we approximate here the GGT $\mathbf{V}^{\text {lnof }}$ expressed in the LNOF along the orbit by a diagonal matrix with $V_{x x}^{\text {lnof }}=V_{y y}^{\text {lnof }}=$ 
$-1372 \mathrm{E}$ and thus $V_{z z}^{\text {lnof }}=2744 \mathrm{E}$. For comparison, $V_{x y}^{\text {lnof }}$ varies between $\pm 0.4 \mathrm{E}, V_{x z}^{\text {lnof }} \pm 8.5 \mathrm{E}$ and $V_{y z}^{\text {lnof }} \pm 1 \mathrm{E}$. In the nadir case, the GGT expressed in the GRF will remain essentially diagonal. As a consequence, we only expect a minor impact of the attitude error on the estimated $V_{y y}$. Assuming a perfectly circular orbit and under the previous hypothesis, the GGT in the GRF for the quasi-inertial mode is given by

$$
\mathbf{V}=\left(\begin{array}{ccc}
686-2058 \cos \left(2 \omega_{\text {lorf }} t\right) & 0 & -2058 \sin \left(2 \omega_{\text {lorf }} t\right) \\
0 & -1372 & 0 \\
-2058 \sin \left(2 \omega_{\text {lorf }} t\right) & 0 & 686+2058 \cos \left(2 \omega_{\text {lorf }} t\right)
\end{array}\right)
$$

In this mode, ${ }^{a t} \delta V_{x x}$ and ${ }^{a t} \delta V_{z z}$ will be dominated by the partial projection of $V_{x z}$. If we note $\sigma_{d \theta_{y}}$ the standard deviation of the angle error $d \theta_{y}$, then the standard deviation of the induced error on the gradient becomes $2\left|V_{x z}\right| \sigma_{d \theta_{y}}=$ $4116 \sigma_{d \theta_{y}}\left|\sin \left(2 \omega_{\text {lorf }} t\right)\right|$ E. With $\sigma_{d \theta_{y}} \approx 1.7 \times 10^{-6}$ rad in our case, the error will have an amplitude of $7 \mathrm{mE}$ modulated at twice the orbital frequency. We therefore expect the attitude error to be a critical issue in the quasi-inertial mode and a major contributor to the noise contaminating both $V_{x x}$ and $V_{z z}$.

\section{Results}

In this section, global gravity field models recovered from gravity gradients in both nadir and quasi-inertial mode are resolved up to degree/order 240 . Both the true errors (the differences between the recovered gravity field coefficients and the input background model, i.e. EIGEN-6C4) and the formal errors (the accompanied standard deviations of the parameters, obtained in the LS adjustment) are analysed.

\subsection{Nadir mode}

Only the gravity gradient in the cross-track direction $V_{y y}$ is measurable in this case. We first investigate the impact of the gradiometer noise by running simulations for 4 different levels of white noise (cases 1 to 4 ). The different cases are summarized in Table 1 and the error degree variances are shown in terms of geoid height in Figure 6(a).

As expected, the gravity field model derived from observations with a noise of $7.0 \mathrm{mE} / \sqrt{\mathrm{Hz}}$ performs worst while the model derived for a noise of $2.5 \mathrm{mE} / \sqrt{\mathrm{Hz}}$ performs best. In between, the performances of the gravity field models are gradually improved when the noise is decreasing. The model 
Table 1: List and attributes of the different simulations run for the nadir case. nom. stands for nominal error level.

\begin{tabular}{lllllll}
\hline & case 1 & case 2 & case 3 & case 4 & case 5 & case 6 \\
\hline grad. noise $(\mathrm{mE} / \sqrt{\mathrm{Hz}})$ & 7 & 5 & 4 & 2.5 & 5 & 2.5 \\
attitude err. & nom. & nom. & nom. & nom. & none & none \\
\hline
\end{tabular}

derived from observations with $2.5 \mathrm{mE} / \sqrt{\mathrm{Hz}}$ noise is about twice better than that from the larger noise of $5 \mathrm{mE} / \sqrt{\mathrm{Hz}}$, especially in the spectral band above degree 50. This is coherent with the levels of input noise. Furthermore, the patterns of the true and formal errors coincide with each other. This indicates that the error propagation of the simulation model is correct. For an identical orbit and timespan, Figure 6(b) shows that the solution derived from the GOCE $V_{y y}$ is slightly better than the CAI gradiometer with a nominal noise (case 2) for degrees larger than 100. The GOCE solution derived from the combination of $V_{x x}, V_{y y}$ and $V_{z z}$ (not plotted here) is even better over degrees larger than 20. We therefore conclude that under the same conditions (orbit and duration), the CAI gradiometer in nadir mode with a noise of $5 \mathrm{mE} / \sqrt{\mathrm{Hz}}$ does not outperform GOCE.

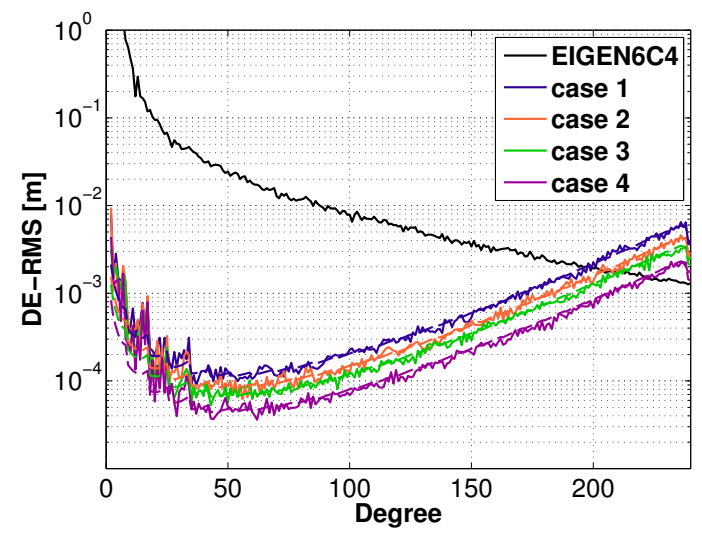

(a)

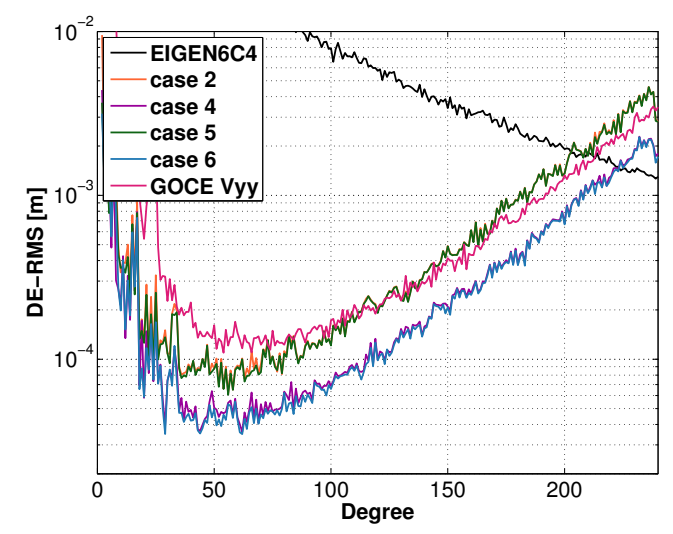

(b)

Figure 6: Error degree variance of the geoid height for the gravitational models derived from 71-day measurements in nadir mode. Solid curves represent the true errors and dashed curves are formal errors. In Figure 6(b) the solution without attitude error (cases 5 and 6) and the model error derived from the GOCE $V_{y y}$ for 71 days of data at the same altitude are plotted for comparison. 
The pyramid representation of the model errors are also shown in Figure 7. We find that the sectorial and near-sectorial coefficients are determined with high precision. This is due to the fact that $V_{y y}$ is approximately oriented in cross-track direction so that the gravity field features in this direction are better mapped.

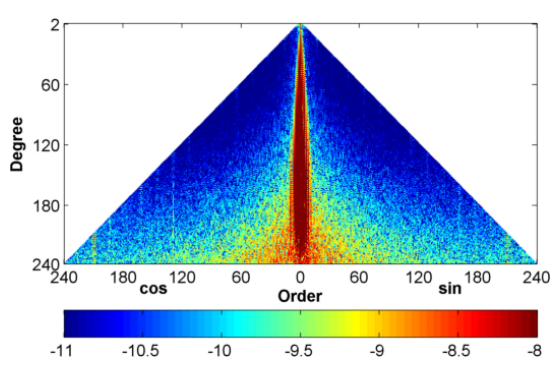

(a) True error case 1

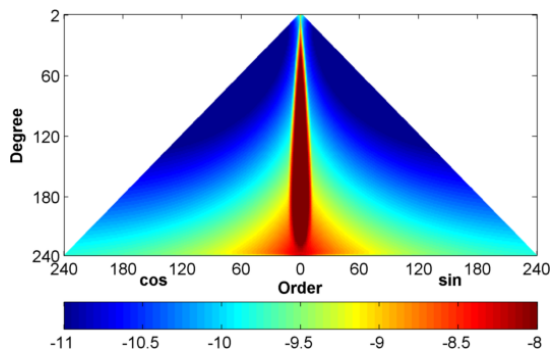

(c) Formal error case 1

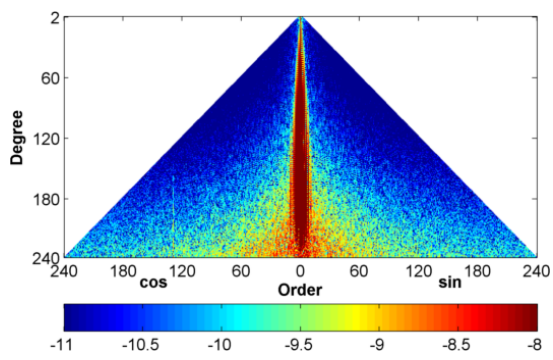

(e) True error case 5

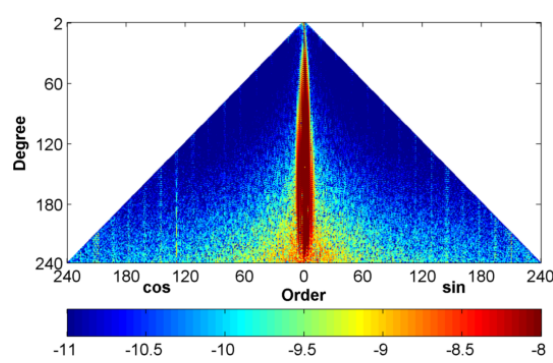

(b) True error case 2

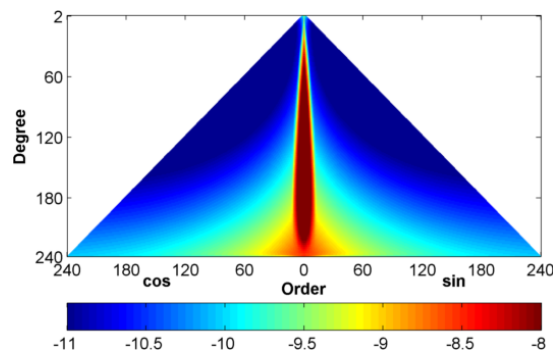

(d) Formal error case 2

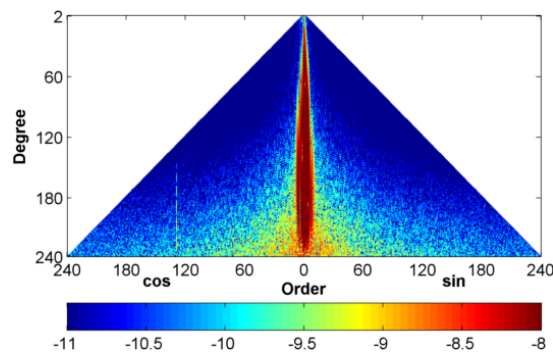

(f) True error case 6

Figure 7: Pyramid representation of relative errors (logarithm scale) of the Stokes coefficients. The left column shows simulations with a nominal gradiometer noise of $5 \mathrm{mE} / \sqrt{\mathrm{Hz}}$ while in the right column the results for a gradiometer noise of $2.5 \mathrm{mE} / \sqrt{\mathrm{Hz}}$ are given. For the first and second row, the computation is made with noisy attitude quaternions while noise-free quaternions are used for the third row.

However, some faint stripes appear in the pyramid representation of the 
true errors at orders of $m=16 \times k, k \in \mathbb{N}$. From the ASD of the post-fit residuals of case 2 and 4, displayed in Figure 8, the peaks at frequencies of multiple (with even numbers) cycles per revolution (cpr) are supposed to be responsible for these stripes since the coefficients at orders $m=16 \times k$ are particularly sensitive to those frequencies. A similar conclusion is also drawn in the processing of GOCE gravity gradients, see Stummer et al. (2011).

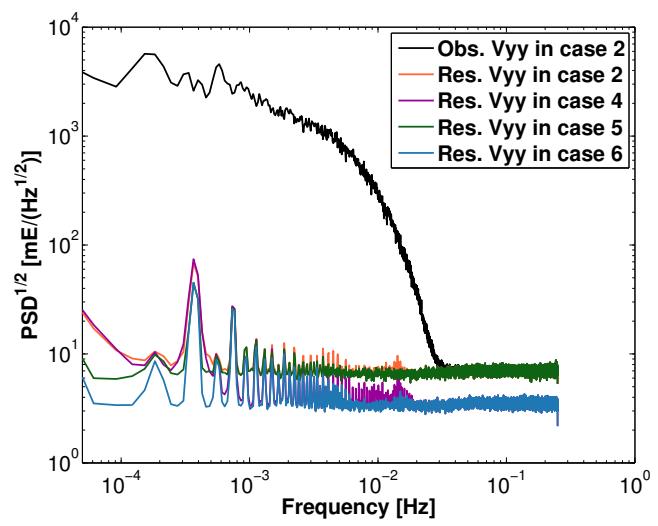

Figure 8: Amplitude spectral density of post-fit residuals in the nadir mode.

To further explore the reason for these stripes, the effect of the quaternion noise is investigated. We generate two new data sets, case 5 and case 6 , with the same noisy $V_{y y}$ as in respectively case 2 and case 4 but with noise-free quaternions. In terms of degree variance (see Figure 6(b)), there is no evident difference between the models (case 2 vs. case 5 , and case 4 vs. case 6 ). But in the pyramid representation of the true errors in Figure 7, most stripes disappear in the solutions where the noise-free quaternions are used. Only one tiny stripe is still visible at the order of about 120 . It is supposed to be caused by the sub-cycle of the repeat ground track orbit which is about eight days. The ASDs of the post-fit residuals are also compared in Figure 8. With the noise-free quaternions, the ASDs of the residuals at the very low frequencies (below about $2 \mathrm{cpr}$ ) are significantly reduced and the amplitude of the peaks at multiples of $2 \mathrm{cpr}$ are slightly attenuated. This agrees with the improvement of the coefficients at orders $m=16 \times k$.

\subsection{Quasi-inertial mode}

In the quasi-inertial mode the three diagonal gravitational gradients are measured. As a consequence, we would expect better results than in the nadir 
mode. Here, the most critical point to investigate is the impact of the error of the estimated GRF attitude. Contrary to the nadir mode, a significant part of the energy of the GGT is located in the off-diagonal elements of the tensor, as outlined in section 4.2.3. In particular, any error in the knowledge of the GRF orientation will introduce a partial projection of $V_{x z}$ on the diagonal gradients $V_{x x}$ and $V_{z z}$, which is much larger than the gradiometer noise.

We carry out two simulations, case 1 and case 2 , for which the gradiometer noise is the same and set to its nominal value, i.e., $5 \mathrm{mE} / \sqrt{\mathrm{Hz}}$. The difference between both cases is on the noise of the gyrometer angular velocity. In case 1 , the angular velocity noise is at its nominal value while in case 2 the noise is divided by 2 . The consequence is twofold: first the error on the attitude quaternions is lower in case 2 and second, the error due to the correction of the centrifugal terms in case 2 is also smaller than in case 1 . For both cases, we derived solutions from each gravitational gradient taken separately (component-wise solution) and a solution combining the three gradients (combined solution).

The error degree variance of the true errors of the estimated spherical harmonic coefficients in terms of geoid height error for both cases are plotted in Figure 9. As expected, the component-wise solutions from $V_{x x}$ and $V_{z z}$ have a larger error compared to the one derived from $V_{y y}$ while the latter compares quite well with the nadir solution (nadir case 2). The solutions derived from $V_{x x}$ and $V_{z z}$ show a similar error which is not surprising since they are contaminated by the same dominant error related to the attitude error and the projection of $V_{x z}$. The regular oscillations on both error are due to the modulation of this error at twice the orbital frequency. The comparison between the solutions of case 1 and case 2 confirms however the slight improvement of case 2 , as can be seen from their respective combined solutions.

The quality difference of the models recovered from $V_{y y}$ on the one hand, and $V_{x x}$ and $V_{z z}$ on the other hand, is even better illustrated in the pyramid representation of the model errors in Figure 10. In this figure, one can see that stripes at orders $m=16 \times k, k \in \mathbb{N}$ degrade significantly the gravitational models derived from $V_{x x}$ and $V_{z z}$, and to a much lower extent from $V_{y y}$. These stripes correspond to the regular oscillations in the degree variance curves in Figure 9. Like in the nadir case, we also observe the presence of peaks at 2 cpr and multiples in the ASDs of the post-fit residuals of $V_{x x}$ and $V_{z z}$ plotted in Figure 11. They correspond to the error contaminating $V_{x x}$ and $V_{z z}$ which is essentially caused by the attitude error and the partial projection of the 


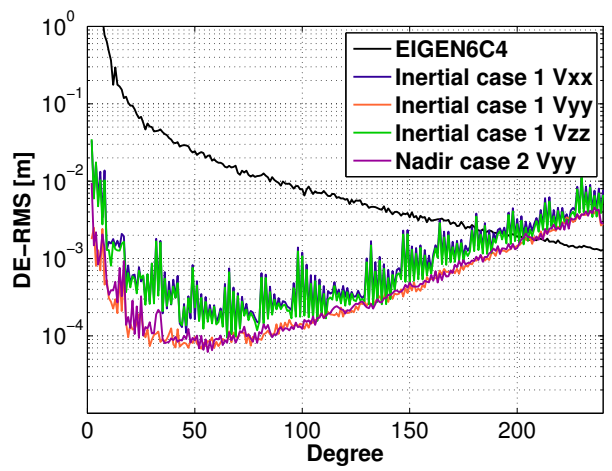

(a) case 1

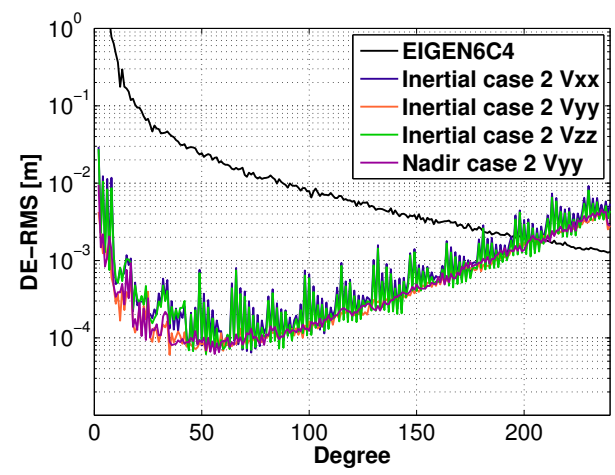

(b) case 2

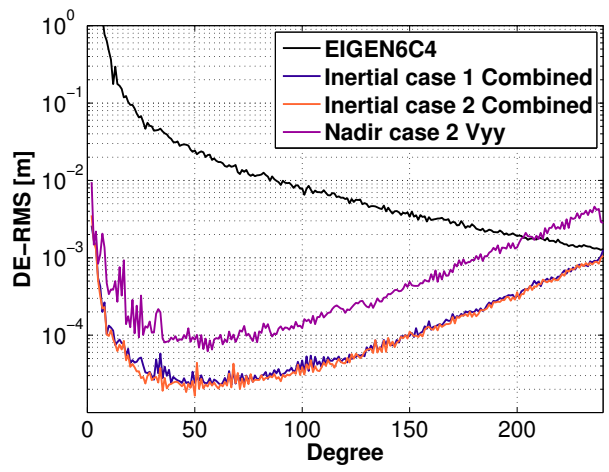

(c) Combined solution for both cases

Figure 9: Degree variances of true errors for the component-wise (Fig.9(a) and 9(b)) and combined (Fig.9(c)) gravity field solutions derived from 71-day measurements in quasiinertial mode. For comparison, the solution in the nadir mode with nominal gradiometer and gyrometer noises (Nadir case $2 V_{y y}$ ) is plotted. 
$V_{x z}$ component also modulated at $2 \mathrm{cpr}$. This specific error on the gradients is directly mapped onto the Stokes coefficients of order $m=16 \times k, k \in \mathbb{N}$. We can thus conclude that these stripes are directly related to the attitude error.

It is noteworthy that $V_{y y}$ is less affected by these stripes. The reason is that we have forced the y-axis of the gradiometer to be always perpendicular to the orbital plane. In this way, we make sure that the projection of the off-diagonal gradients on $V_{y y}$ due to the attitude error always have a weak amplitude, just like in the nadir mode. This is the reason why the GRF must follow the rotation of the orbital plane: if we keep a truly inertial attitude then the y-axis would slowly rotate with respect to the orbital plane and eventually be parallel to it, like $V_{x x}$ or $V_{z z}$ initially. Accordingly, the noise on $V_{y y}$ due the attitude error would become similar to the one on the 2 other gradients, with the same notable stripes.

A first idea in order to mitigate these stripes would be to have a more accurate and precise attitude determination for the quasi-inertial case than for the nadir case. We will see however that a simple processing can largely reduce these stripes. We introduce empirical parameters to absorb the lowfrequency contributions that are contained in the post-fit residuals. More specifically, a drift and a bias term are added to the functional model and estimated in an arc-wise manner. The length of the arc has been fixed by trial and error to 15 minutes. These parameters are estimated together with the Stockes coefficients. Following this method, the gravity field solutions of case 1 are re-computed and shown in Figure 12. This figure also includes the plots of the ASD of the corresponding post-fit residuals. The amplitude of the peaks at $2 \mathrm{cpr}$ and multiples are dramatically reduced for all gradients, as compared to the previous situation. As expected, the error level and oscillations of the degree variances for the component-wise solutions of $V_{x x}$ and $V_{z z}$ and the combined solution are also attenuated. This is also reflected in the pyramid distribution of the true errors in Figure 10 where the stripes almost totally disappeared.

It can be pointed out that the post-fit residual ASDs drop below $1 \mathrm{mE} / \sqrt{\mathrm{Hz}}$ for frequencies smaller than $2 \times 10^{-4} \mathrm{~Hz}$, which -according to Figure 4 - is approximately the magnitude of the time-variable gravitational signals in this frequency domain. Yet, this does not allow to detect the time-variable signal. First because the arc-wisely estimated empirical parameters tend to absorb the signal and noise at low frequencies (at least lower than $\frac{1}{15 \times 60} \approx$ $1.1 \times 10^{-3} / \mathrm{Hz}$ ) and this effect increases as the frequency decreases. There- 


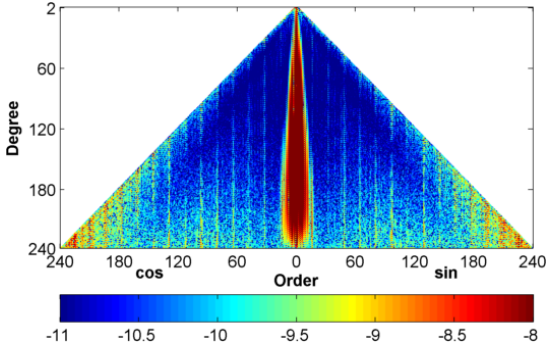

(a) $V_{x x}$ in case 1

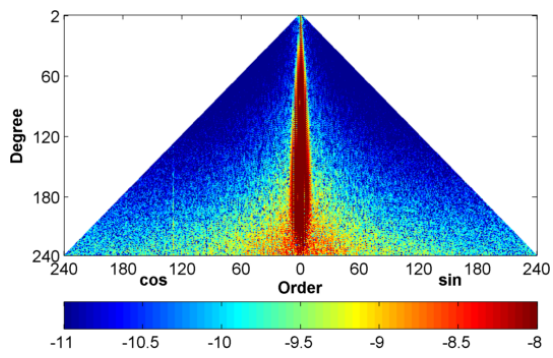

(c) $V_{y y}$ in case 1

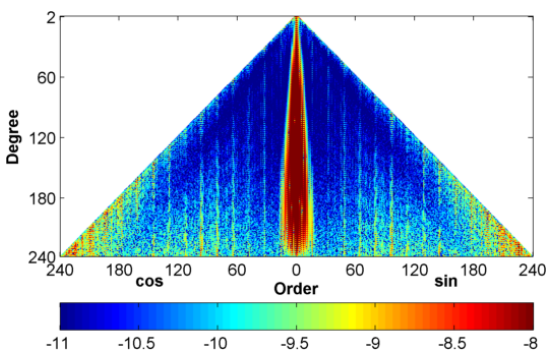

(e) $V_{z z}$ in case 1

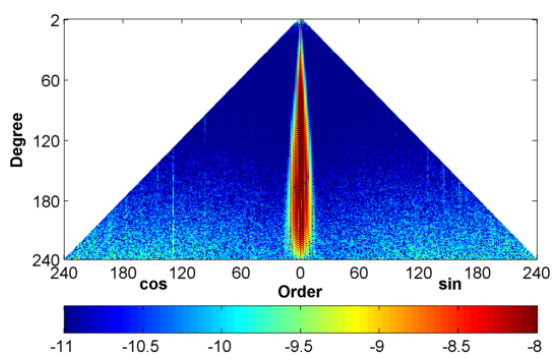

(g) combined solution in case 1

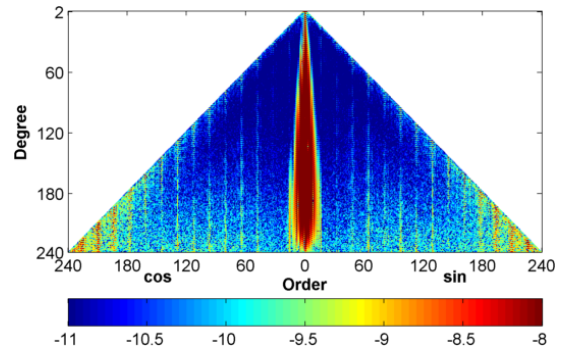

(b) $V_{x x}$ in case 2

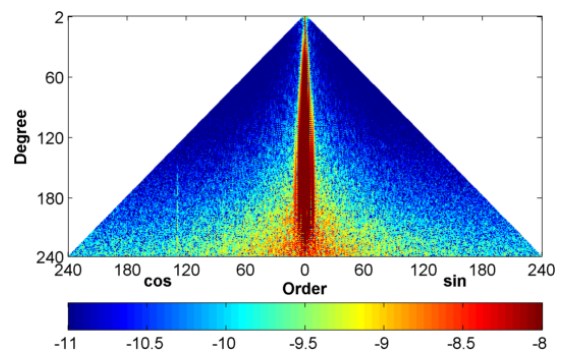

(d) $V_{y y}$ in case 2

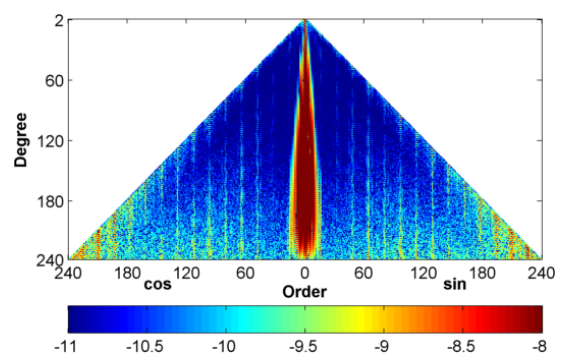

(f) $V_{z z}$ in case 2

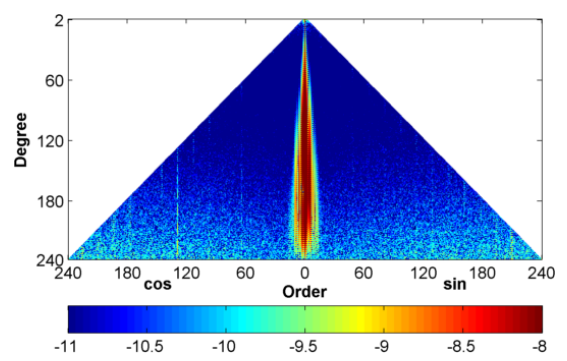

(h) combined solution in case 2

Figure 10: Pyramid representation of true errors (logarithm scale) of the Stokes coefficients. The left column shows component-wise and combined solutions of case 1 while the right column shows results of case 2 . 


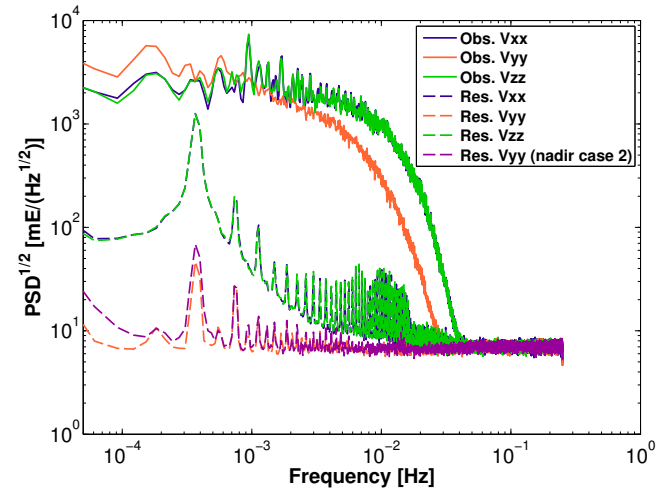

(a) case 1

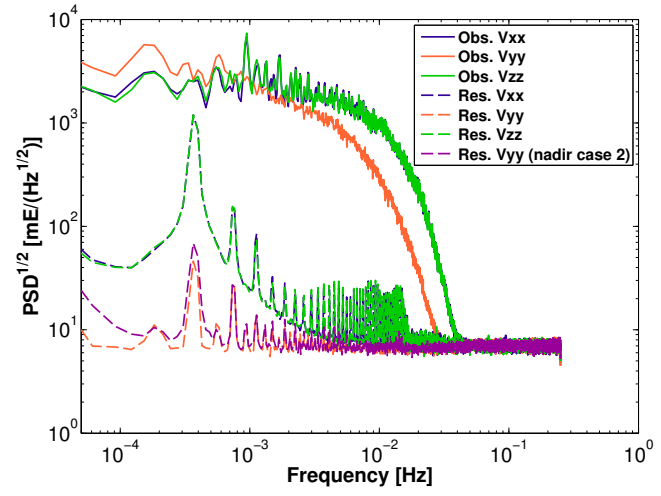

(b) case 2

Figure 11: Amplitude spectral density of the post-fit residuals in the quasi-inertial mode for case 1 and 2 .

fore this signal would not be captured by the gravitational model. Second, the gravitational signal at frequencies smaller than $1 \mathrm{cpr} \approx 1.8 \times 10^{-4} / \mathrm{Hz}$ is related to spatial variations of the field whose wavelengths, when sensed along the orbit, is greater than 1 revolution. It is therefore a complex mix of spatially and / or temporally aliased gravitational signal, also modulated by variations of the orbit altitude. In our case, where only a static gravity field is considered, we can see from the example of $V_{x x}$ in Figure 12 that the absorption of these low frequencies does not degrade the recovery of the field.

Although white noise is assumed for the gradiometer, we see that the spectral shape of the post-fit residuals exhibits a slight colour behaviour, especially in the low-frequency part. To further improve the estimation of the gravity field solutions, we run a second analysis where the variance/covariance matrix is taken into account to model the correlation of the observations. We notice a tiny improvement for degrees smaller than 100, however the error above degree 100 remains at the same level as before.

\section{Discussion}

We analyse now to what extent the CAI gradiometer concept can outperform GOCE when assuming a nominal gradiometer noise of $5 \mathrm{mE} / \sqrt{\mathrm{Hz}}$. To this end, we extrapolate the error on these models from 71 days to 8 months assuming the error is purely stochastic and reduces as $\sqrt{t_{i}}$ where $t_{i}$ is the time of integration. To gain an idea of the GOCE solution error, we first 

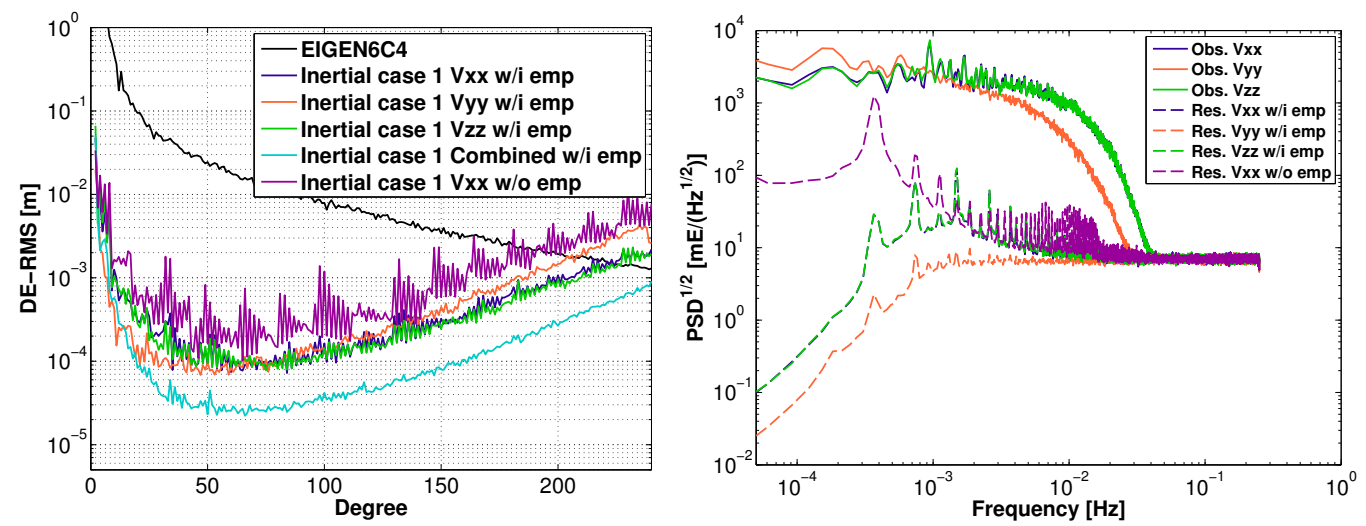

Figure 12: Degree variances of the true errors of the gravity field model recovered in case 1 (left) and amplitude spectral density of the post-fit residuals (right) when empirical parameters are included in the LS adjustment. For comparison, the same quantities are plotted when no empirical parameters are considered for $V_{x x}$.

compute a gravity model based on the three diagonal gravitational gradients of the whole GOCE mission period (November 2009 - October 2013, about 47 months). The least-squares adjustment is performed for GOCE data in the same way as for our simulations. Then, we compare it to 2 different reference gravity models: on the one hand, the EIGEN-6C4 model used for the synthesis of the gravitational gradients in the simulation and, on the other hand, the GOCO05S (Mayer-Guerr, 2015). Since the comparison concerns only the contribution of the gradiometer, we have not taken into account the high-low satellite-to-satellite tracking data used to recover the low degrees of the gravity model. The results are shown in Figure 13 in terms of geoid height degree variance. We can note two bumps on the GOCE solution when it is compared to EIGEN-6C4. One appears in the range between d/o 100 and 130; the other one is visible between d/o 170 and 200. These bumps are due to the background model errors. This appears clearly when we compare our derived gravity field solutions to a satellite combined solution, i.e., GOCO05s. The bumps disappear in the GOCE solution but appear in the quasi-inertial solution based on EIGEN-6C4. Besides these two bumps, we can make the following comments:

- As already mentioned in section 5.2, for identical conditions, the solution in the quasi-inertial mode is significantly better than the nadir mode one, especially above d/o 50. Additionally, the nadir mode with 

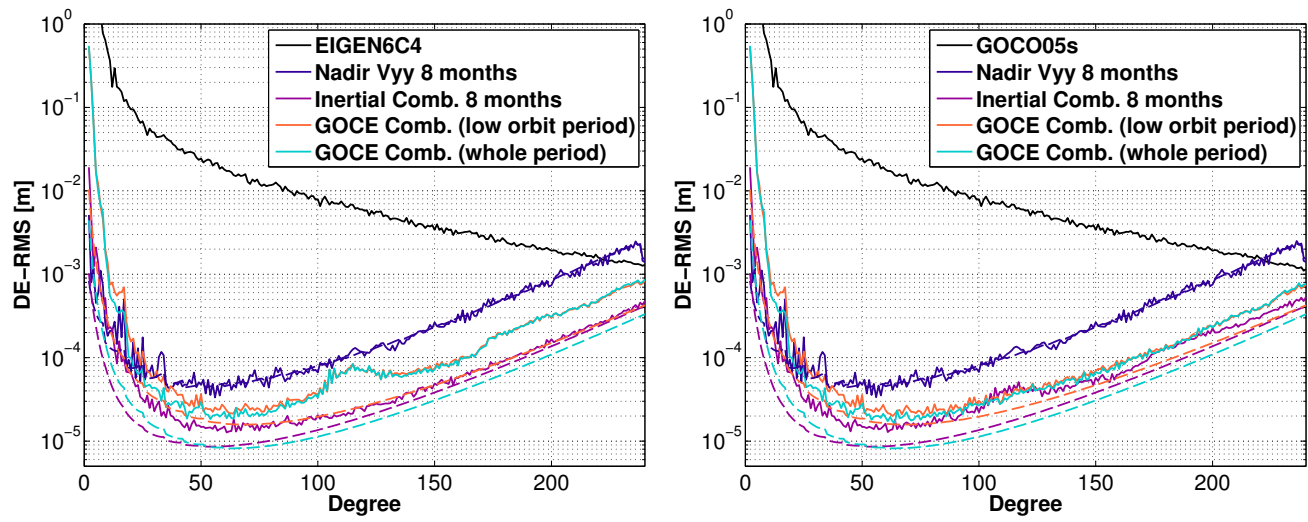

Figure 13: Extrapolation of the error of the gravity field solutions to 8 months for the CAI gradiometer and comparison to the error of the GOCE gradiometer-only solution. For GOCE, the whole period combined solution is based on the gravitational gradients of the whole mission period while the low orbit period solution correspond the 8 last months during which the satellite was at an altitude equal or lower than $239 \mathrm{~km}$.

a nominal noise does not allow to outperform GOCE under identical conditions (orbit and measurement timespan), see section 5.1. As a consequence, the nadir mode should be discarded in favour of the quasi-inertial mode.

- The 8-month quasi-inertial solution is better than the GOCE solution for the whole mission period. We can thus conclude that an 8-month mission at an altitude of $239 \mathrm{~km}$ and using a 3-axis CAI gradiometer with a nominal white noise of $5 \mathrm{mE} / \sqrt{\mathrm{Hz}}$ in the quasi-inertial mode would outperform the full GOCE mission and eventually yields a more precision gravity field model. Nonetheless, it should be borne in mind in this comparison that the GOCE satellite was most of its lifetime at a higher altitude than $239 \mathrm{~km}$, about 3 months at $239 \mathrm{~km}$ and 5 months lower than $239 \mathrm{~km}$. This fact shows however that it is technically possible to fly a satellite at an altitude as low as $239 \mathrm{~km}$ for a duration of 8 months.

- For a fairer comparison, we have also plotted the GOCE solution for the 8 months during which the satellite was at an altitude equal or lower than $239 \mathrm{~km}$ (see Figure 13). Again, the 8-month quasi-inertial solution at a constant altitude yields a slightly better solution despite 
the fact that the 8-month GOCE solution is partly based on gravity gradients measured at a lower altitude.

Among the assumptions made in this study, the attitude control requirements and the quasi-inertial mode seem particularly challenging, especially at such a low altitude where the atmospheric drag may cause variations on the satellite angular velocity greater than $1 \mu \mathrm{rad} / \mathrm{s}$. Inertial pointing satellites are rather uncommon although control feedback strategies to maintain such a pointing have been studied (Lovera and Astolfi, 2005) and robust solutions using only magnetorquers have been put forward, see e.g. (Celani, 2015) and (Celani, 2016). The MICROSCOPE space mission (Touboul et al., 2012) launched in April 2016 is an example of a satellite partly operated in inertial pointing with a specification on the angular velocity stability smaller than $10^{-9} \mathrm{rad} / \mathrm{s}$ (Touboul et al., 2012). However such an inertial pointing stability is achieved after a long in-orbit calibration and at an altitude of 720 $\mathrm{km}$, where the atmospheric drag is considerably smaller than at the altitude we assumed in our simulation.

\section{Summary, conclusions and recommendations}

Recent developments in light-pulse atom interferometry have opened the way to the design of new high-accuracy and high precision inertial sensors, in particular when operated in a microgravity environment where the operating conditions are optimal. Used in an accelerometer configuration and in pairwise assemblies, atom interferometers constitute the backbone of the space gravitational gradiometer concept introduced in this article. A nominal white noise of $5 \mathrm{mE} / \sqrt{\mathrm{Hz}}$ is expected for the CAI gradiometer. Its performance and ability to recover the Earth's gravitational field have been investigated via closed-loop simulations assuming realistically degraded ancillary data (position, angular velocity, gradiometer noise and attitude quaternions).

Because the atom interferometer architecture requires to limit the angular velocity perpendicular to the measurement axis to $1 \mu \mathrm{rad} / \mathrm{s}$, only two operating modes are possible for the considered CAI gradiometer concept: the nadir mode and the quasi-inertial mode. In the nadir mode only the $V_{y y}$ component, along the across-track direction, is measured. With one observed gradient, our simulations show that the nadir mode does not allow to clearly outperform GOCE. The results are more promising in the case of the quasi-inertial mode where the gradiometer can measure the three diagonal 
components of the GGT. To outperform the whole GOCE mission in term of geoid accuracy, we conclude that a CAI gradiometer operated in quasiinertial mode for at least 8 months, on a circular orbit and at an altitude of $240 \mathrm{~km}$ is required. Of course, this simple scenario is just an indication and further studies are required to find the best trade-off between the orbit altitude, the mission life-time and the technical limitations.

Although the quasi-inertial mode proved to be the best operating mode for the CAI gradiometer considered in this study, keeping such a pointing seems technically challenging. Therefore we recommend to investigate whether it is possible to modify the design of the CAI gradiometer so that the 3 diagonal elements of the GGT can be measured in the nadir mode. In other words, an improved interferometer architecture that allows the atoms to interfere in an almost uniform rotation reference frame is highly desirable. A solution for the compensation of the Earth rotation in ground based set-ups has already been implemented (Lan et al., 2012). It consists in repeatedly counter-rotating the reference mirror using tip-tilt actuators. However, implementing such a solution would require in our case large tilt chirps of the mirror, if a single mirror is to be used. This is not readily implementable in the case of our interleaved interrogation geometry. As an alternative, we are currently studying an architecture based on a set of mirrors with pre-adjusted tilts, which would allow to cancel the effect of rotation at the orbital frequency.

With the 3 diagonal elements of the GGT measured in the nadir mode, better performances are expected, particularly thanks to the $V_{z z}$ component which would be estimated directly along the radial direction. Furthermore, the off-diagonal gradients would keep a small amplitude, leading to a much smaller impact of the attitude error determination on the error of the estimated gradients.

\section{Acknowledgements}

This study was supported by the European Space Agency through Contract No. 4000112677/14/NL/MP. The authors would like to thank all the collaborators of the project, in particular Baptiste Battelier, Andrea Bertoldi, Sven Herrmannn, Merle Cornelius, Naceur Gaaloul, Dennis Becker, Ernst Rasel and Norman Guerlebeck. Comments and feedbacks from Christian Siemes were greatly appreciated. K. Douch, J. Müller, C. Schubert and H. $\mathrm{Wu}$ also acknowledge the Collaborative Research Center (SFB) 1128 geo-Q, funded by the Deutsche Forschungsgemeinschaft (DFG). C. Schubert would 
like to thank Sven Abend for comments and acknowledges financial support from "Niedersächsisches Vorab" through the "Quantum and Nano- Metrology (QUANOMET)" initiative within the project QT3.

\section{References}

Abend, S., Gebbe, M., Gersemann, M., et al., Nov 2016. Atom-chip fountain gravimeter. Phys. Rev. Lett. 117, 203003.

URL https://link.aps.org/doi/10.1103/PhysRevLett.117.203003

Aguilera, D., Ahlers, H., Battelier, B., et al., 2014. Ste-questtest of the universality of free fall using cold atom interferometry. Classical and Quantum Gravity 31 (11), 115010.

Antoine, C., 2007. Rotating matter-wave beam splitters and consequences for atom gyrometers. Phys. Rev. A 76, 033609.

Arimondo, E., Ertmer, W., Schleich, W. P., Rasel, E. M. (Eds.), 2009. Lightpulse atom interferometry. Vol. 168 of Atom Optics and Space Physics. Proceedings of the International School of Physics "Enrico Fermi", J. M. Hogan, D. M. S. Johnson, M. A. Kasevich.

Barrett, B., Antoni-Micollier, L., Chichet, L., et al., 2016. Dual matter-wave inertial sensors in weightlessness. Nat. Comm. 7, 13786.

Berg, P., Abend, S., Tackmann, G., et al., Feb 2015. Composite-light-pulse technique for high-precision atom interferometry. Phys. Rev. Lett. 114, 063002.

URL https://link.aps.org/doi/10.1103/PhysRevLett.114.063002

Bock, H., Jäggi, A., Meyer, U., et al., 2011. GPS-derived orbits for the goce satellite. Journal of Geodesy 85 (11), 807.

URL http://dx.doi.org/10.1007/s00190-011-0484-9

Bordé, C., 2004. Quantum Theory of Atom-Wave Beam Splitters and Application to Multidimensional Atomic Gravito-Inertial Sensors. Gen. Rel. Grav. 36, 475.

Carraz, O., Siemes, C., Massotti, L., et al., 2014. A spaceborne gravity gradiometer concept based on cold atom interferometers for measuring Earth's 
gravity field. Microgravity Science and Technology 26 (3), 139-145.

URL http://dx.doi.org/10.1007/s12217-014-9385-x

Celani, F., 2015. Robust three-axis attitude stabilization for inertial pointing spacecraft using magnetorquers. Acta Astronautica 107, 87-96.

URL http://www.sciencedirect.com/science/article/pii/ S0094576514004615

Celani, F., 2016. Spacecraft attitude stabilization using magnetorquers with separation between measurement and actuation. Journal of Guidance, Control, and Dynamics 39 (9), 2184-2191.

URL https://doi.org/10.2514/1.G001804

Dickerson, S. M., Hogan, J. M., Sugarbaker, A., et al., 2013. Multiaxis Inertial Sensing with Long-Time Point Source Atom Interferometry. Phys. Rev. Lett. 111, 083001.

Dobslaw, H., Bergmann-Wolf, I., Dill, R., et al., 2015. The updated ESA Earth System Model for future gravity mission simulation studies. Journal of Geodesy 89 (5), 505-513.

URL http://dx.doi.org/10.1007/s00190-014-0787-8

Eicker, A., Forootan, E., Springer, A., et al., 2016. Does GRACE see the terrestrial water cycle intensifying? Journal of Geophysical Research: Atmospheres 121 (2), 733-745, 2015JD023808.

URL http://dx.doi.org/10.1002/2015JD023808

Elsaka, B., Raimondo, J.-C., Brieden, P., et al., 2014. Comparing seven candidate mission configurations for temporal gravity field retrieval through full-scale numerical simulation. Journal of Geodesy 88 (1), 31-43.

URL http://dx.doi.org/10.1007/s00190-013-0665-9

Förste, C., Bruinsma, S., Abrikosov, O., et al., 2014. EIGEN-6C4-the latest combined global gravity field model including GOCE data up to degree and order 1949 of GFZ Potsdam and GRGS Toulouse. In: EGU General Assembly Conference Abstracts. Vol. 16. p. 3707.

Freier, C., Hauth, M., Schkolnik, V., et al., 2016. Mobile quantum gravity sensor with unprecedented stability. J. of Phys.: Conf. Ser. 723, 012050. 
Gruber, T., Team, 2014. e2. motion: Earth system mass transport mission (square)concept for a next generation gravity field missionfinal report of project satellite gravimetry of the next generation (nggm-d). Tech. rep., Deutsche Geodätische Kommission der Bayerischen Akademie der Wissenschaften, Reihe B Angewandte Geodäsie Heft Nr. 318, München.

Hofmann-Wellenhof, B., Moritz, H., 2006. Physical geodesy. Springer Science \& Business Media.

Hu, Z.-K., Sun, B.-L., Duan, X.-C., et al., 2013. Demonstration of an ultrahigh-sensitivity atom-interferometry absolute gravimeter. Phys. Rev. A 88, 043610.

Kovachy, T., Asenbaum, P., Overstreet, C., et al., 2015a. Quantum superposition at the half-metre scale. Nature 528, 530.

Kovachy, T., Hogan, J., Sugarbaker, A., et al., 2015b. Matter wave lensing to picokelvin temperatures. Phys. Rev. Lett. 114, 143004.

Kusche, J., Klemann, V., Bosch, W., 2012. Mass distribution and mass transport in the Earth system. Journal of Geodynamics 5960, 1-8, mass Transport and Mass Distribution in the System Earth.

URL http://www.sciencedirect.com/science/article/pii/ S0264370712000440

Lan, S.-Y., Kuan, P.-C., Estey, B., et al., 2012. Influence of the coriolis force in atom interferometry. Phys. Rev. Lett. 108, 090402.

Lévèque, T., Gauguet, A., Michaud, F., et al., 2009. Enhancing the Area of a Raman Atom Interferometer Using a Versatile Double-Diffraction Technique. Phys. Rev. Lett. 103, 080405.

Louchet-Chauvet, A., Farah, T., Bodart, Q., et al., 2011. The influence of transverse motion within an atomic gravimeter. New J. Phys. 13, 065025.

Lovera, M., Astolfi, A., 2005. Global magnetic attitude control of inertially pointing spacecraft. Journal of guidance, control, and dynamics 28 (5), 1065-1072.

Mayer-Guerr, T., 2015. The combined satellite gravity field model GOCO05s. In: EGU General Assembly Conference Abstracts. Vol. 17. p. 12364. 
McGuirk, J. M., Foster, G. T., Fixler, J. B. o., Feb 2002. Sensitive absolutegravity gradiometry using atom interferometry. Phys. Rev. A 65, 033608. URL https://link.aps.org/doi/10.1103/PhysRevA.65.033608

Müntinga, H., Ahlers, H., Krutzik, M., et al., 2013. Interferometry with Bose-Einstein Condensates in Microgravity. Physical Review Letters 110, 093602.

Pail, R., Bingham, R., Braitenberg, C., et al., 2015. Science and user needs for observing global mass transport to understand global change and to benefit society. Surveys in Geophysics 36 (6), 743-772.

URL http://dx.doi.org/10.1007/s10712-015-9348-9

Panet, I., Flury, J., Biancale, R., et al., 2013. Earth system mass transport mission (e.motion): A concept for future Earth gravity field measurements from space. Surveys in Geophysics 34 (2), 141-163.

URL http://dx.doi.org/10.1007/s10712-012-9209-8

Peters, A., Chung, K. Y., Chu, S., 1999. Measurement of gravitational acceleration by dropping atoms. Nature 400, 849-852.

URL http://dx.doi.org/10.1038/23655

Rosi, G., Sorrentino, F., Cacciapuoti, L., et al., 2014. Precision measurement of the newtonian gravitational constant using cold atoms. Nature 510 (7506), 518-521.

Roura, A., Apr 2017. Circumventing heisenberg's uncertainty principle in atom interferometry tests of the equivalence principle. Phys. Rev. Lett. 118, 160401.

URL https://link.aps.org/doi/10.1103/PhysRevLett.118.160401

Roura, A., Zeller, W., Schleich, W. P., 2014. Overcoming loss of contrast in atom interferometry due to gravity gradients. New Journal of Physics 16 (12), 123012.

URL http://stacks.iop.org/1367-2630/16/i=12/a=123012

Rudolph, J., Herr, W., Grzeschik, C., et al., 2015. A high-flux BEC source for mobile atom interferometers. New J. Phys. 17, 065001.

Rummel, R., Yi, W., Stummer, C., 2011. GOCE gravitational gradiometry. Journal of Geodesy 85 (11), 777. 
Sneeuw, N., Van Gelderen, M., 1997. The polar gap. In: Geodetic boundary value problems in view of the one centimeter geoid. Springer, pp. 559-568.

Stummer, C., Fecher, T., Pail, R., 2011. Alternative method for angular rate determination within the GOCE gradiometer processing. Journal of Geodesy 85 (9), 585-596.

URL http://dx.doi.org/10.1007/s00190-011-0461-3

Tackmann, G., Berg, P., Schubert, C., et al., 2012. Self-alignment of a compact large-area atomic Sagnac interferometer. New J. Phys. 14, 015002.

Tapley, B. D., Bettadpur, S., Watkins, M. o., 2004. The gravity recovery and climate experiment: Mission overview and early results. Geophysical Research Letters 31 (9), n/a-n/a, 109607. URL http://dx.doi.org/10.1029/2004GL019920

Torge, W., Müller, J., 2012. Geodesy. Walter de Gruyter.

Touboul, P., Métris, G., Lebat, V., et al., 2012. The MICROSCOPE experiment, ready for the in-orbit test of the equivalence principle. Classical and Quantum Gravity 29 (18), 184010.

URL http://stacks.iop.org/0264-9381/29/i=18/a=184010

Zupan, E., Saje, M., 2011. Integrating rotation from angular velocity. Advances in Engineering Software 42 (9), 723-733.

URL http://www.sciencedirect.com/science/article/pii/ S0965997811001177 